University of Wollongong

Research Online

Faculty of Informatics - Papers (Archive)

Faculty of Engineering and Information

Sciences

2010

\title{
Fast quality-guided flood-fill phase unwrapping algorithm for three- dimensional fringe pattern profilometry
}

Ke Chen

University of Wollongong

Jiangtao Xi

University of Wollongong, jiangtao@uow.edu.au

Yanguang Yu

University of Wollongong, yanguang@uow.edu.au

Joe F. Chicharo

University of Wollongong, chicharo@uow.edu.au

Follow this and additional works at: https://ro.uow.edu.au/infopapers

Part of the Physical Sciences and Mathematics Commons

\section{Recommended Citation}

Chen, Ke; Xi, Jiangtao; Yu, Yanguang; and Chicharo, Joe F.: Fast quality-guided flood-fill phase unwrapping algorithm for three-dimensional fringe pattern profilometry 2010.

https://ro.uow.edu.au/infopapers/3519

Research Online is the open access institutional repository for the University of Wollongong. For further information contact the UOW Library: research-pubs@uow.edu.au 


\title{
Fast quality-guided flood-fill phase unwrapping algorithm for three-dimensional fringe pattern profilometry
}

\author{
Abstract \\ A fast quality-guided flood-fill phase unwrapping algorithm is proposed for real-time 3D Fringe Pattern \\ Profilometry (FPP) system. The proposed approach consists of three steps. First, based on the phase \\ maps acquired by phase shift profilometry (PSP) techniques, a quality map is generated according to the \\ phase variance adjacent pixels on the wrapped phase map. According to the quality map, the phase map \\ is divided into several parts which are categorised as either rapid phase changing areas or smooth phase \\ changing areas. Then quality-guided flood-fill phase unwrapping algorithm is applied to rapid phase \\ changing areas and non-guided path-following algorithm is used in the smooth phase changing area. The \\ proposed approach is much faster than the conventional non-guided path-following algorithm, and it is \\ more robust than the non-guided path-following algorithm. Experiments are carried out to verify the \\ performance.

\section{Disciplines} \\ Physical Sciences and Mathematics

\section{Publication Details} \\ K. Chen, J. Xi, Y. Yu \& J. F. Chicharo, "Fast quality-guided flood-fill phase unwrapping algorithm for three- \\ dimensional fringe pattern profilometry," in Optical Metrology and Inspection for Industrial Applications, \\ 2010, pp. 1-9.
}




\title{
Fast Quality-guided flood-fill phase unwrapping algorithm for three-dimensional Fringe Pattern Profilometry
}

\author{
Ke Chen, Jiangtao Xi, Yanguang Yu, Joe F. Chicharo \\ School of Electrical, Computer \& Telecommunications Engineering, University of Wollongong, \\ Australia;
}

\begin{abstract}
A fast quality-guided flood-fill phase unwrapping algorithm is proposed for real-time 3D Fringe Pattern Profilometry (FPP) system. The proposed approach consists of three steps. First, based on the phase maps acquired by phase shift profilometry (PSP) techniques, a quality map is generated according to the phase variance adjacent pixels on the wrapped phase map. According to the quality map, the phase map is divided into several parts which are categorised as either rapid phase changing areas or smooth phase changing areas. Then quality-guided flood-fill phase unwrapping algorithm is applied to rapid phase changing areas and non-guided path-following algorithm is used in the smooth phase changing area. The proposed approach is much faster than the conventional non-guided path-following algorithm, and it is more robust than the non-guided path-following algorithm. Experiments are carried out to verify the performance.
\end{abstract}

Keywords: Phase unwrapping, Fringe Pattern profilometry (FPP), Three-dimension measurement

\section{INTRODUCTION}

Fringe Pattern Profilometry (FPP) has been an active area of research as an effective technology for non-contract 3D shape measurement. A typical FPP system consists of a digital projector, a CCD camera and a computer. The core idea of FPP system is that, the digital projector generates a group of image patterns and projects them onto the object surface to be measured [2]. Due to the variance of the surface shape, the image patterns are distorted which are acquired by the CCD camera. The distorted images carry the information of the 3D surface shape and are analysed by the computer, yielding the surface shape of the object [1][2][3]. A class of popular approaches for FPP are based on phase detection (PD), where images patterns are sinusoidal or periodic. With PD, the deformed projected fringe patterns can be modelled as the result of phase modulation of the original fringe patterns. Both fringe patterns can be described by phase maps, and the 3D shape can be retreaved based on analysis of the phase maps. Therefore, most existing PD based approaches consist of the following steps (1) acquire the fringe patterns (2) extract the phase maps, and (3) reconstruct the 3D shapes. A major problem associated with the second step is that the extracted phase maps are wrapped into the range from $-\pi$ to $\pi$. In other words, in contrast to the true phase $\phi$, the extracted phase $\phi_{r}$ becomes $\phi_{r}=\phi \pm 2 m \pi$, where $m$ is an integer making $-\pi<\phi_{r} \leq \pi$. Obviously, when $\phi$ varies on the phase map across the points $\pm \pi, \pm 3 \pi, \pm 5 \pi, \ldots$ etc, the wrapped $\phi_{r}$ exhibits jumps or drops of $2 \pi$, causing discontinuity in the wrapped phase maps. In order to obtain the $3 \mathrm{D}$ shapes, the true phase maps are required and thus must be recovered from the wrapped phase maps. Such a process is called phase unwrapping which is a critical step in the PD based FPP approaches.

Although phase unwrapping is rather simple in its principle, its implementation in practice is a challenging task. In the cases that the true phase is continuous, slow varying and not affected by noise, phase unwrapping can be carried out by adding or subtracting $2 \pi$ to the result of unwrapped phase map when the wrapped one reaches $-\pi$ or $\pi$. However, when the acquired wrapped maps are corrupted by unwanted noise or disturbance, or the true phase maps have discontinuities larger than $2 \pi$, the task becomes very difficult as wrong decision may be made regarding the addition or subtraction of $2 \pi$. In 3D shape measurement with PD based FPP, the later situations are rather common as the FPP systems are inevitably influenced by various noise or disturbance, and for objects with a complex and discontinues surface shapes, the true phase map are discontinuous in nature. Consequently, phase unwrapping under these situations has been an active area of research during the past two decades.

Optical Metrology and Inspection for Industrial Applications, edited by Kevin Harding, Peisen S. Huang, Toru Yoshizawa, Proc. of SPIE Vol. 7855, 78550X - (C) 2010 SPIE · CCC code: 0277-786X/10/\$18 · doi: 10.1117/12.870232 
Several phase unwrapping algorithms have been proposed to recover the true phase map. These algorithms can be classified as three categories: (1) global algorithms, (2) region growing algorithms, and (3) path-following algorithms [2]. The global algorithms utilize a global function to formulate the unwrapping algorithm [8]. Algorithms in this class are robust but computationally intensive, that is they can accurately unwrap complex phase maps but take too much time. The region growing algorithms divide phase map into several regions. Each region is unwrapped individually and then merged with its adjoin regions to form a whole unwrapped phase map [6]. In contrast to global algorithms, region growing algorithms are less computational intensive but also less robust.

The path-following algorithms carry out phase unwrapping along a continuous path on the phase map. Compared with global and region growing algorithms, path-following algorithms are less computational complex. There are three classes of path following algorithms: (1) fixed path-following methods [8], (2) branch-cut methods [5], and (3) qualityguided methods [1]. The simplest methods among these three classes are fixed path-following method. The fixed pathfollowing methods undertake phase unwrapping along a fixed path, and thus are very fast, but they are not able to recover the true phase if there is a jump or drop of phase more than $2 \pi$ between two adjacent pixels (phase jump) or phase noise. In contrast to fixed path-following methods, branch-cut methods are more robust. With the branch-cut methods, phase jumps in a phase map are detected and marked by curves, referred to the cuts. The unwrapping path is prohibited to go over the cuts and hence wrong phase unwrapping decisions can be limited. With quality-guided methods, the unwrapping path is determined based on the pixels quality [1], and thus the methods are more robust than other pathfollowing algorithms.

Two path-following phase unwrapping algorithms are commonly used in FPP systems, which are the non-guided pathfollowing which belongs to fixed path-following methods and the quality-guided flood-fill which belongs to quality guided methods [1][2][3]. The non-guided path-following is simple and fast. However, this approach is not suitable for complex phase maps. In contrast to non-guided path-following, quality-guided flood-fill method is suitable for complex phase maps, but it suffers from the problem of long time to complete a phase unwrapping task [2][3][7][8], thus making itself not suitable for real-time measurement. Consequently, it is necessary to find a new method with improved performance in terms of both robustness, i.e. unwrapping accuracy, and speed.

In this paper, we propose a new unwrapping approach called fast quality-guided flood-fill. The idea of the proposed approach is to combine non-guided path-following method and quality-guided flood-fill method together. In the proposed approach, the wrapped phase map is divided into two types of areas according to a quality map, including the rapid phase changing areas and smooth phase changing areas. Then quality-guided flood-fill method is applied to the rapid phase changing area and non-guided path-following method to the smooth phase changing area. Experiments show that both speed and robustness can be ensured with the proposed approach.

The following of this paper is organised as follows. In Section 2, existing approaches are introduced. In Section 3, proposed approach is shown. In Section 4, experiments results are demonstrated. Finally, the conclusions are drawn.

\section{EXISTING APPROACHES}

\subsection{Quality maps}

As mentioned above, the challenges associated with the phase unwrapping lie in the complexity of the unwrapped phase map. When the wrapped phase maps are smooth and slow varying, it is quite easy to recover the true phase by nonguided path-following approach. However, for the wrapped phase with sharp changes or discontinuities, the tasks are very difficult. Based on such a scenario people introduced a metric called quality map to evaluate unwrapped phase maps in terms of the difficulty associated with their unwrapping. Let us use $s(x, y)$ to denote the light intensity of a fringe pattern, where $x$ and $y$ are pixel index numbers. The corresponding wrapped phase map will be $\phi_{r}(x, y)$, and accordingly the quality map will take $Q(x, y)$, with its element determined according to a quality parameter of each pixel on the wrapped phase map. The quality parameter at a certain pixel can be measured by phase variance between adjoin pixels [2][3][8]. The phase variance between adjoin pixels at pixel $(x, y)$ can be calculated by [3][8]: 


$$
\left.\Delta \phi(x, y)=\frac{1}{4}\left[\phi_{r}(x, y)-\phi_{r}(x, y-1)|+| \phi_{r}(x, y)-\phi_{r}(x, y+1)|+| \phi_{r}(x, y)-\phi_{r}(x-1, y)|+| \phi_{r}(x, y)-\phi_{r}(x+1, y)\right]\right]
$$

where, $\phi(x, y)$ is the wrapped phase at pixel $(x, y)$, and $\Delta \phi(x, y) \in[0,2 \pi]$. The quality parameter at the pixel $(x, y)$ can be determined by the following:

$$
Q(x, y)=1-\frac{\Delta \phi(x, y)}{2 \pi}
$$

Obviously, $0 \leq Q(x, y) \leq 1$, and the smaller the phase variance $\Delta \phi(x, y)$, the larger the quality parameter $Q(x, y)$. By calculating the quality parameters for all the pixels on the phase map using Equation (1) and (2), we are able to obtain $Q(x, y)$ for all the pixels. If we use an image of the same size as the phase map to represent $Q(x, y)$ with the brightest (white) for 0 and the darkest for 1 , the resulting image is called quality map.

\subsection{Non-guided path-following algorithm}

In the family of path-following approaches, the simplest one is non-guided path-following method proposed by Schafer and Oppenheim [9]. The path of unwrapping is simply vertical or horizontal from one border of the phase map to the other. Let us assume that the unwrapping direction is horizontal and the wrapped phase map size is $M \times N$. The unwrapped phase $\phi$ of pixel $\left(x_{m}, y_{n}\right)$ can be calculated as follows:

$$
\phi\left(x_{m}, y_{n}\right)=\left\{\begin{array}{clcc}
\phi\left(x_{m-1}, y_{n}\right)+\Delta \phi_{r}\left(x_{m}, y_{n}\right)+2 \pi & \text { if } & \Delta \phi_{r}\left(x_{m}, y_{n}\right) \leq-\pi \\
\phi\left(x_{m-1}, y_{n}\right)+\Delta \phi_{r}\left(x_{m}, y_{n}\right) & \text { if } & -\pi<\Delta \phi_{r}\left(x_{m}, y_{n}\right)<\pi \\
\phi\left(x_{m-1}, y_{n}\right)+\Delta \phi_{r}\left(x_{m}, y_{n}\right)-2 \pi & \text { if } & \Delta \phi_{r}\left(x_{m}, y_{n}\right) \geq \pi
\end{array}\right.
$$

where $\Delta \phi_{r}\left(x_{m}, y_{n}\right)=\phi_{r}\left(x_{m}, y_{n}\right)-\phi_{r}\left(x_{m-1}, y_{n}\right), m \in[1, M], n \in[0, N]$.

$\phi_{r}\left(x_{m}, y_{n}\right)$ is the wrapped phase of pixel $\left(x_{m}, y_{n}\right)$.

As shown in Equation (3), the unwrapping process will start at pixel $\left(x_{0} y_{0}\right)$ and unwrap all the pixels in a same row. Then, the process goes to pixel $\left(x_{0} y_{1}\right)$ and unwrap all the pixels of that row. The process will continue until all rows are unwrapped.

\subsection{Quality-guided flood-fill algorithm}

As described above, non-guided path-following approaches carry out phase unwrapping on row-by-row or column-bycolumn path basis on the phase map. In case there is a phase jump or noise in the wrapped phase map, the methods fail to recover the true phase and these errors will propagate to the following processed pixels. In order to solve the problem, quality-guided flood-fill algorithm is proposed [2][3][8]. The details of quality-guided flood-fill algorithm are shown below. In order to clearly describe the process, let us assume that we have a cursor with its position indicating the pixel on which phase unwrapping is currently carried out.

Step 1. Firstly, find out the pixel with the highest quality parameter on the quality map $Q(x, y)$. We assume that phase value of this point on the wrapped phase map is the same as the true phase (or we are able to unwrap this point to yield the true phase value). Put the cursor to this point and mark the point as "unwrapped", and then start the unwrapping process as follows.

Step 2. Check the quality parameters of the four pixels surrounding the cursor, that is, the pixels to the left and the right, the ones above and below. Find out the pixel with the highest quality parameter and move the cursor to the pixel and then unwrap the phase value.

Step 3. Let $\phi_{r}(n)$ indicates the wrapped phase of current cursor point, $\phi_{r}(n-1)$ and $\phi(n-1)$ indicate the wrapped phase and unwrapped phase of previous point respectively. The unwrapped phase of current cursor point $\phi(n)$ can be determined as following: 


$$
\phi(n)=\left\{\begin{array}{ccc}
\phi(n-1)+\Delta \phi_{r}(n)+2 \pi & \text { if } & \Delta \phi_{r}(n) \leq-\pi \\
\phi(n-1)+\Delta \phi_{r}(n) & \text { if } & -\pi<\Delta \phi_{r}(n)<\pi \\
\phi(n-1)+\Delta \phi_{r}(n)-2 \pi & \text { if } & \Delta \phi_{r}(n) \geq \pi
\end{array}\right.
$$

where $\Delta \phi_{r}(n)=\phi_{r}(n)-\phi_{r}(n-1)$. Upon completion of the above, the pixel is marked as "unwrapped".

Step 4. Check the surrounding pixels around unwrapped pixels and select the pixel with the highest quality parameter and move the cursor to the pixel, then repeat Step 3 until all pixels have been unwrapped.

The advantage of quality-guided flood-fill is that phase unwrapping is always carried out following the paths of high quality pixels, that is, with relatively small variance from pixel to the next. As phase unwrapping errors are more likely occur at pixels with lower quality, such a quality-guided approach will be able to improve the robustness of phase unwrapping.

\section{PROPOSED APPROACH}

Our proposed approach combines non-guided path-following and quality-guided flood-fill together. The details of the approach are shown below:

Firstly, the quality map is generated according to quality parameters as described in Section 2.1. The pixels on the quality map are classified into two categories by means of a threshold associated with the quality parameter $Q_{t h}(x, y)$. The pixels with quality parameter larger than the threshold are categorized as the high quality pixels, and the remaining as the low quality pixels. The threshold $Q_{t h}(x, y)$ should be neither too big nor too small, otherwise either phase unwrapping robustness or speed will be degraded.

Secondly, we mark the quality map by rectangles in such a way that only the high quality pixels are inside these rectangles. As the result, the quality map will be divided into different type of areas. The areas inside the rectangles are characterized by smooth phase variation and hence referred to as high quality areas, and the parts outside the rectangles are called low quality areas due to rapid phase variation.

Thirdly, after rapid and smooth phase changing areas are identified, a start point will be selected. The start point can be any high quality pixel on the boundary of the quality map. Then, the order for phase unwrapping process will be determined by following criteria to keep unwrapping path continuous: (1) The areas closer to the start point will be unwrapped earlier than the areas with larger distance to start the point. (2) If there are several areas have a similar distance to the start point, the high quality areas will be unwrapped earlier than the low quality areas. Then, the phase unwrapping process will be commenced by following this order. The high quality areas and low quality areas are unwrapped using the non-guided path following and quality-guided flood-fill algorithms described above respectively.

Finally, the results of all the areas are merged together to yield a true phase map.

\section{EXPERIMENTS}

In order to demonstrate the proposed approach and compare it with existing phase unwrapping algorithms, we applied them to images acquired by a 3D shape measurement system in our laboratory. In the experiment system, the sinusoidal fringe patterns are generated by a HITACHI CP-X260 digital projector, and a Duncan Tech MS3100-RGB 3CCD digital camera is utilized to capture the images. The digital camera is placed on top of the projector with a distance of $350 \mathrm{~mm}$ between their lenses. The distance between the reference plane and camera lens is $1295 \mathrm{~mm}$. The three-step phaseshifting profilometry (3-PSP) has been used to acquire phase information and calculate surface height of object.

In the first experiment, we used a milk bottle as the target and Figure 1(a) shows the camera capture of measured object. Figure 1(b) (c) (d) shows the three-step PSP fringe images. The resolution of image is $416 \times 369$ pixels. The phase shift

between each step is $\frac{2 \pi}{3}$. The wrapped phase map obtained using the 3-PSP is shown in Figure 1(e). Then we convert 
the phase map into the quality map using Equation (1) and (2) shown in Figure 2 (a). Both considering the unwrapping robustness and speed, a threshold of quality parameter at $Q_{t h}(x, y)=0.20$ is applied to categorize high and low quality areas. The start point $\left(x_{0}, y_{0}\right)$ is at upper right corner of the quality map. In Figure 2(b). The areas marked by alphabets are high quality areas and the areas marked by numbers are low quality areas. According to the criteria of ordering, the order of those areas for phase unwrapping process is A, B, C, D, E, F, 1, G, H, I, 2.

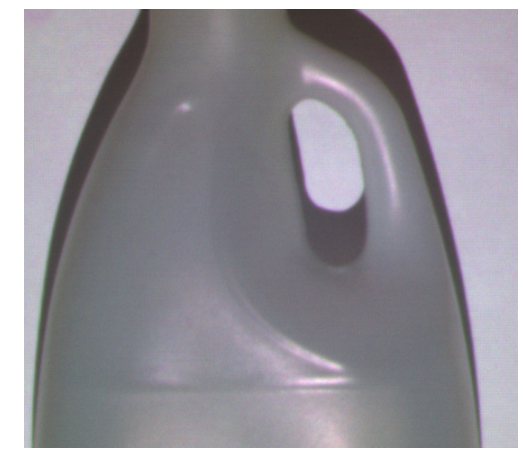

(a)

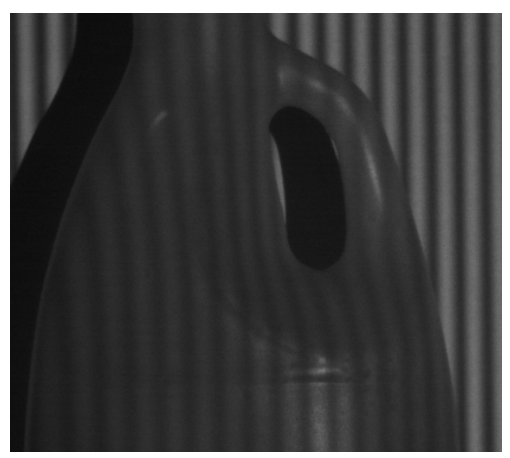

(c)

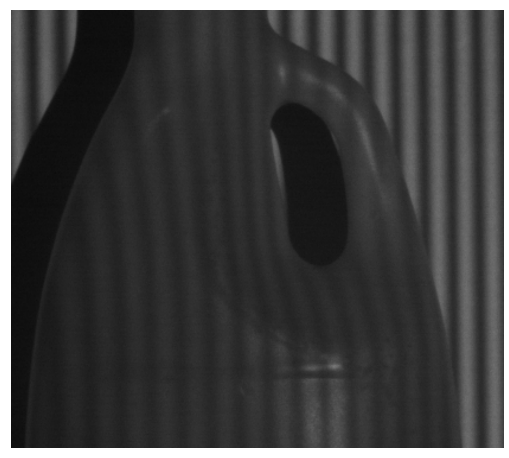

(b)

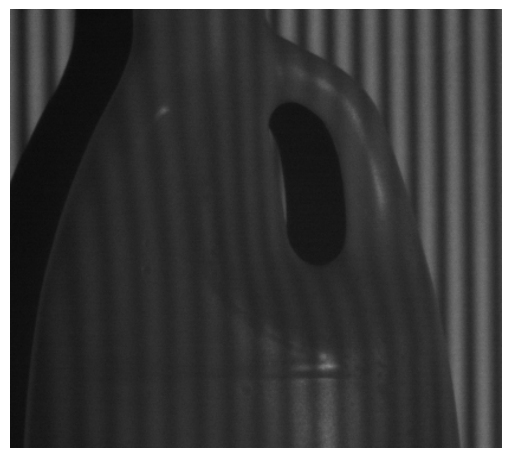

(d)

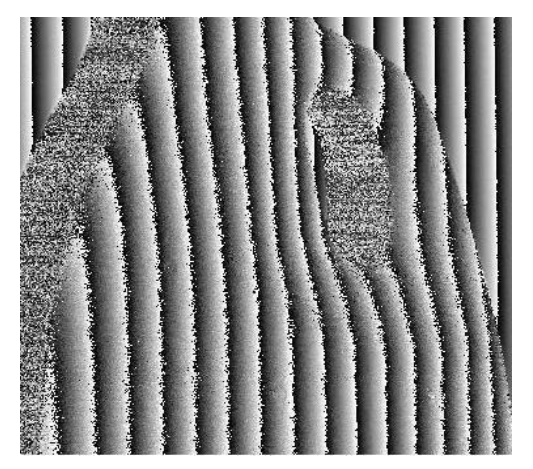

(e)

Figure 1. (a) The camera capture of measured object. (b) (c) (d) Three-step PSP fringe images with starting phases at 0 , $\frac{2 \pi}{3}, \frac{4 \pi}{3}$ respectively. (e) Wrapped phase map. 


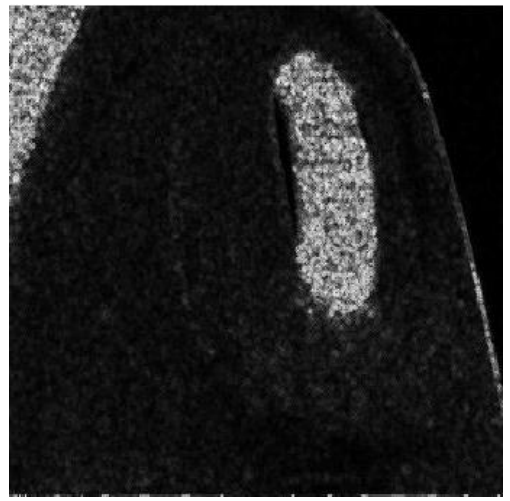

(a)

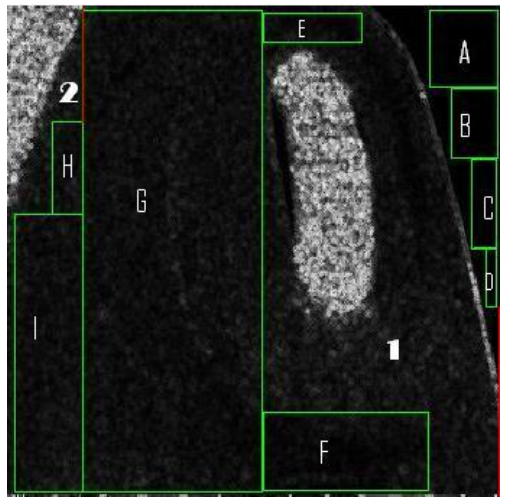

(b)

Figure 2. (a) The quality map for conventional quality-guided method. (b) The quality map for proposed method.

For comparison purpose, we reconstructed the shape of measured object using the phase maps recovered by non-guided path-following algorithm, the proposed algorithm and quality-guided flood-fill algorithm respectively, and the results are shown in Figures 3 (a) (b) and (b). We can see that, in terms of smoothness of the reconstructed shape which reflects the phase unwrapping robustness, the proposed approach is close to quality-guided flood-fill algorithm and much better than the non-guided path-following algorithm.

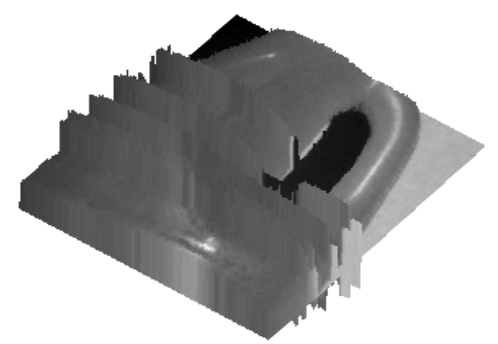

(a)

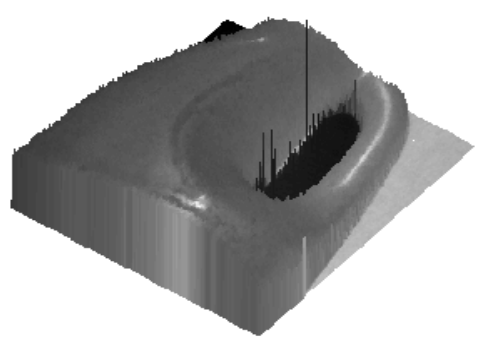

(b)

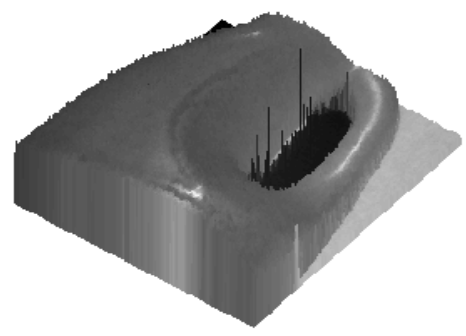

(c)

Figure 3. Three-dimensional reconstruction results using (a) Non-guided path-following algorithm, (b) Proposed algorithm, (c) Quality-guided flood-fill algorithm.

We also carried out experiments on a plaster palm model. Figure 4(a) is the camera capture of measured object. Figure 4(b) shows the quality map. Figure 4(c) shows the smooth and rapid phase changing areas in the quality map. We choose the start point to be the upper right corner of the quality map. The reconstructed shapes for this set of experiments are shown in Figure 5. 
Based on the experiments we can say that the proposed approach is able to achieve robustness performance close to that of quality-guided flood-fill algorithm, but with much less computations and hence can be much faster. In order to compare the computational burden, let us consider the number of phase unwrapping operations in Equation (3) (4) and the number of comparison operations. For a phase map of the size $M \times N$, the non-guided flood-fill algorithm requires $M N$ phase unwrapping operations only, but the quality-guided flood-fill algorithm requires the same number of unwrapping operations and additional $C(M, N)$ comparisons, given by the following:

$$
\frac{1}{4} M^{3} N+\frac{1}{4} M N^{3}-M-N-2 \leq C(M, N) \leq \frac{1}{9} M^{3} N^{3}+\frac{5}{18} M^{2} N^{2}-\frac{4}{3} M N-\frac{N}{3}+2
$$

From Equation (5), we can see that the computational burden associated with non-guided path-following algorithm is much lower than that of quality-guided flood-fill algorithm. When the proposed approach is applied to the two experiments, the whole phase map is divided into 9 and 11 high quality areas with the total size of $226 \times 369$ and $270 \times 369$ respectively, and 2 low quality areas with the total size of $190 \times 369$ and $146 \times 369$ respectively. With regard to experiments results, the proposed approach is able to significant reduce the computation. Table 1 give comparison of the time consumption for each algorithm.

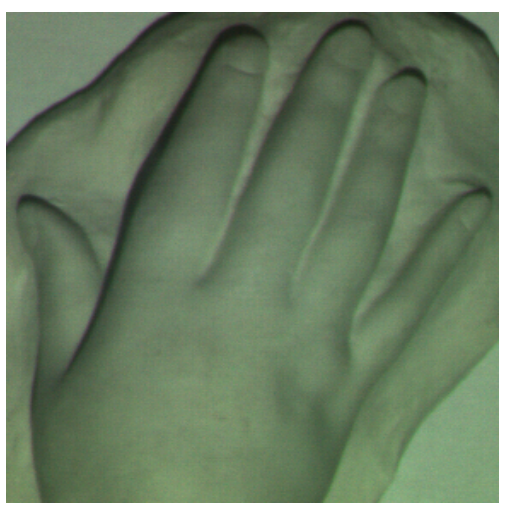

(a)

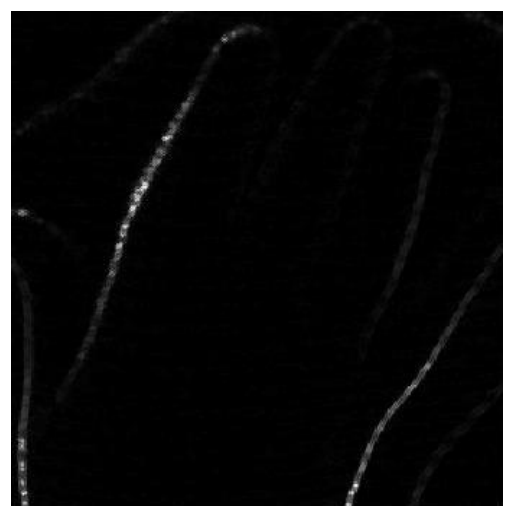

(b)

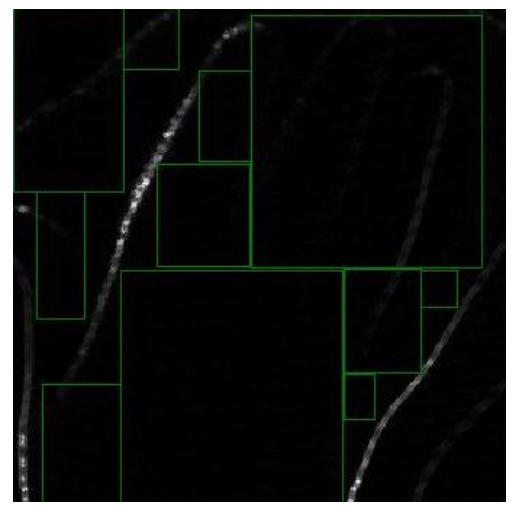

(c)

Figure 4. (a) Camera capture of a plaster palm model. (b) The quality map for conventional quality-guided method. (c) The quality map for proposed phase unwrapping method. 


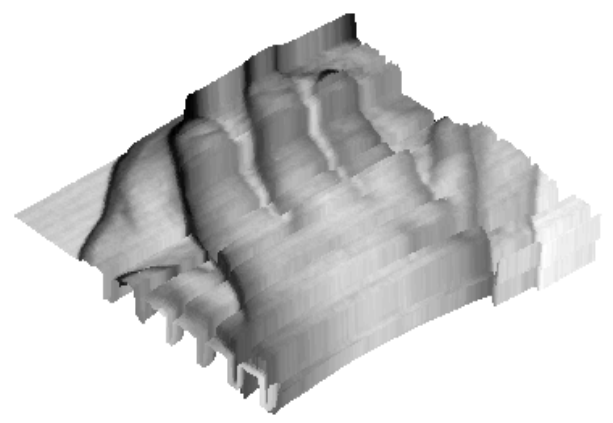

(a)

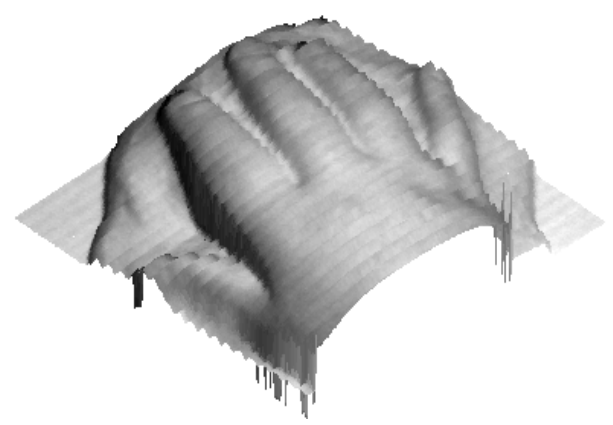

(b)

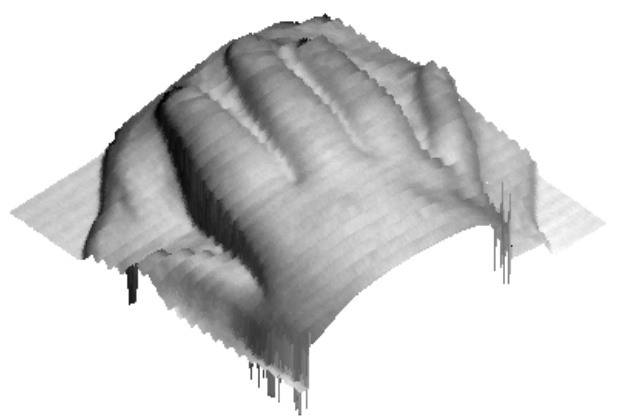

(c)

Figure 5. (a) Three-dimensional reconstruction results using (a) Non-guided path-following algorithm, (b) Proposed algorithm (c) Quality-guided flood-fill algorithm.

Table.1. Comparison of Phase Unwrapping Time of Different Algorithms

\begin{tabular}{lccc} 
& $\begin{array}{c}\text { Non-guided } \\
\text { path-following }\end{array}$ & $\begin{array}{c}\text { Quality-guided } \\
\text { flood-fill }\end{array}$ & $\begin{array}{c}\text { Proposed } \\
\text { method }\end{array}$ \\
\hline Unwrapping time for bottle & $0.0484 \mathrm{~s}$ & $280.3368 \mathrm{~s}$ & $35.6432 \mathrm{~s}$ \\
Unwrapping time for palm & $0.0467 \mathrm{~s}$ & $279.5323 \mathrm{~s}$ & $23.3452 \mathrm{~s}$
\end{tabular}

The computation time was acquired by a Deltacom desktop (Intel Q9400@2.66GHz, 4 G memory; the image resolution is $416 \times 369$ pixels

\section{CONCLUSIONS}

We presented a fast quality-guided flood-fill phase unwrapping algorithm for three-dimensional FPP system based on the combination of two conventional phase unwrapping algorithms. The quality map was generated according to phase variance between adjacent pixels of the wrapped phase map. This quality map was divided into two types of areas, namely smooth and rapid phase changing areas. A non-guided path-following algorithm was applied to the smooth phase changing areas and a quality-guided flood-fill phase unwrapping algorithm was applied in the rapid phase changing areas. As demonstrated by theoretical analysis and experiment results, the proposed approach is characterized by much less computational burden but is able to achieve the same level of $3 \mathrm{D}$ measurement robustness in comparison with conventional quality-guided flood-fill algorithm. 


\section{REFERENCES}

[1] Zhang, S., Li, X. and Yau, S., "Multilevel quality-guided phase unwrapping algorithm for real-time threedimensional shape reconstruction," Applied Optics, 46, 50-57 (2007).

[2] Zappa, E. and Busca, G., "Comparison of eight unwrapping algorithms applied to Fourier-transform profilometry," Optics and Lasers in Engineering, 46, 106-116 (2008).

[3] Su, X. and Chen, W., "Reliability-guided phase unwrapping algorithm: a review," Optics and Lasers in Engineering, 42, 245-261 (2004).

[4] He, X., Kang, X., Tay, C., Quan, C. and Shang, H., "Proposed algorithm for phase unwrapping," Applied Optics, 41, 7422-7428 (2002).

[5] Salfity, M. F., Ruiz, P.D., Huntley, J. M., Graves, M. J., Cusack, R. and Beauregard, D.A., "Branch cut surface placement for unwrapping of under sampled three-dimensional phase data: application to magnetic resonance imaging arterial flow mapping," Applied Optics, 34, 5100-5108 (1995).

[6] Baldi, A., "Phase unwrapping by region growing," Applied Optics, 42, 2498-2505 (2003).

[7] Herraez, M., Boticario, J. G., Lalor, M. J. and Burton, D. R., "Agglomerative clustering-based approach for two-dimensional phase unwrapping," Applied Optics, 44, 1129-1140 (2005).

[8] Ghiglia, D. C. and Pritt, M. D., "Two-Dimensional Phase Unwrapping-Theory, Algorithms, and Software," John Wiley \& Sons, INC. New York, 100-310 (1998).

[9] Schafer, R. W., and Oppenheim, A. V., "Digital Signal Processing," Prentice-Hall, Englewood Cliffs, New Jersey, 507-511 (1975). 